\title{
Alimentos funcionais: conhecimento e consumo por usuários de restaurante self-service em capital do nordeste brasileiro
}

\author{
Functional foods: knowledge and consumption by self-service restaurant users in \\ northeast brazilian capital
}

\begin{abstract}
Alimentos funcionales: conocimimento y consumo por usuarios de restaurante self-service en capital del nordeste brasileño
\end{abstract}

Maria Francisca do Nascimento Costa'; Beatriz Cordeiro de Araújo²; Maísa Guimarães Silva Primo³; Thaís Rodrigues Nogueira ${ }^{4}$; Gilmara Péres Rodrigues ${ }^{5 *}$.

\section{RESUMO}

Objetivo: Descrever o conhecimento e consumo de alimentos funcionais por usuários de restaurante selfservice da capital piauiense. Métodos: Trata-se de estudo transversal descritivo, conduzido com 161 indivíduos, de ambos os sexos, idade de 20 a 59 anos. Os usuários foram investigados quanto à definição de alimentos funcionais. A dieta habitual foi avaliada por aplicação de um questionário de frequência alimentar, adaptado para alimentos funcionais, com as categorias de consumo: habitual, não habitual, raramente consumido e nunca consumido. Os dados obtidos foram analisados por estatística descritiva com auxílio do software IBM SPSS Statistics. O estudo foi aprovado por Comitê de Ética em Pesquisa. Resultados: A amostra, com média de idade de $38,6 \pm 9,0$ anos, apresentou maioria masculina $(57,8 \%)$, com ensino superior completo $(73,3 \%)$. Desta, apenas $36,6 \%$ dos indivíduos definiram corretamente a terminologia "alimentos funcionais", em contradição ao esperado para escolaridade elevada como determinante do conhecimento e qualidade alimentar. A dieta habitual caracterizou-se por baixa ingestão semanal de frutas, hortaliças, cereal integral, leguminosas, óleos insaturados, peixes, oleaginosas, chás e especiarias, sendo insuficiente, portanto, em compostos antioxidantes, anti-inflamatórios e anticarcinogênicos, entre os quais se destacam: resveratrol, licopeno, organossulforetos, catequinas, gingerol, curcumina e ômega 3. Conclusão: Conclui-se que a população de adultos ativos participante deste estudo possui conhecimento inadequado sobre alimentos funcionais, os quais não estão incluídos em sua alimentação habitual.

Palavras-Chave: Alimentos Funcionais, Dieta, Doença Crônica.

\begin{abstract}
Objective: To describe the knowledge and consumption of functional foods for self-service restaurant users in the capital of Piauí. Methods: This was a cross-sectional study, conducted with 161 individuals of both sexes, aged from 20 to 59 years. Users were investigated regarding the definition of functional foods. The usual diet was evaluated using a food frequency questionnaire, adapted for functional foods, with consumption categories: habitual, not habitual, rarely consumed and never consumed. The data were analyzed by descriptive statistics using IBM SPSS Statistics software. The study was approved by the Research Ethics Committee. Results: The sample, with mean age of $38.6 \pm 9.0$ years, presented male majority $(57.8 \%)$ and complete higher education (73.3\%). Of this, only $36.6 \%$ of the individuals correctly defined "functional foods", in contradiction to what was expected for high schooling as a determinant of knowledge and food quality. The
\end{abstract}

1 Centro Universitário UNINOVAFAPI, Teresina, Piauí, Brasil.

2 Universidade Federal do Piauí, Teresina, Piauí, Brasil.

${ }^{3}$ Hospital Universitário da Universidade Federal do Piauí, Teresina, Piauí, Brasil.

${ }^{4}$ Universidade Cândido Mendes, Rio de Janeiro, Rio de Janeiro, Brasil.

${ }^{5}$ Departamento de Nutrição, Universidade Federal do Piauí, Teresina, Piauí, Brasil.

*E-mail: gilmaraperes@ufpi.edu.br 
usual diet was characterized by a low weekly intake of fruits, vegetables, whole grains, legumes, unsaturated oils, fish, oilseeds, teas and spices, being therefore insufficient antioxidant, anti-inflammatory and anticarcinogenic compounds, among which include: resveratrol, lycopene, organosulfurants, catechins, gingerol, curcumin and omega 3. Conclusion: It is concluded that the active adult population participating in this study has inadequate knowledge about functional foods, which are not included in their usual diet.

Key words: Functional Foods, Diet, Chronic Disease.

\section{RESUMEN}

Objetivo: Describir el conocimiento y consumo de alimentos funcionales de usuarios de restaurante self service de la capital piauiense. Métodos: Se trata de un estudio transversal, conducido con 161 individuos, de ambos sexos, edad de 20 a 59 años. Los usuarios fueron investigados en cuanto a la definición de alimentos funcionales. La dieta habitual fue evaluada por aplicación de un cuestionario de frecuencia alimentaria, adaptado para alimentos funcionales, con las categorías de consumo: habitual, no habitual, raramente consumido y nunca consumido. Los datos obtenidos fueron analizados por estadística descriptiva con ayuda del software IBM SPSS Statistics. El estudio fue aprobado por el Comité de Ética en Investigación. Resultados: La muestra, con una media de edad de 38,6 \pm 9,0 años, presentó mayoría masculina (57,8\%) y enseñanza superior completa (73,3\%). De esta, sólo el $36,6 \%$ de los individuos definieron correctamente los "alimentos funcionales", en contradicción a lo esperado para escolaridad elevada como determinante del conocimiento y de la calidad alimentaria. La dieta habitual se caracterizó por una baja ingesta semanal de frutas, hortalizas, cereal integral, leguminosas, aceites insaturados, pescados, oleaginosas, tés y especias, siendo insuficiente, por lo tanto, en compuestos antioxidantes, anti-inflamatorios y anticarcinogénicos, entre los cuales se destacan: resveratrol, licopeno, organosulforetos, catequinas, gingerol, curcumina y omega 3. Conclusión: Se concluye que la población de adultos activos participante de este estudio posee conocimiento inadecuado sobre alimentos funcionales, los cuales no están incluidos en su alimentación habitual.

Palabras clave: Alimentos Funcionales, Dieta, Enfermedad Crónica.

\section{INTRODUÇÃO}

Alimento funcional é definido pela Secretaria de Vigilância Sanitária, do Ministério da Saúde do Brasil, na Portaria no 398 da RDC (Resolução da Diretoria Colegiada) no 18/99, como um tipo de alimento ou componente alimentar que, quando consumido como parte da dieta habitual, além das funções nutritivas básicas, é capaz de produzir efeitos metabólicos e/ou fisiológicos e/ou benéficos à saúde, sendo seguro para consumo humano sem supervisão médica (BRASIL, 1999).

Esses efeitos metabólicos e/ou fisiológicos, desde a terminologia inicial Foods for Specified Health Use ou FOSHU, atribuída aos alimentos funcionais em 1980 no Japão, têm despertado o interesse de muitos pesquisadores em todo o mundo, na tentativa de conhecer as propriedades funcionais e sua aplicabilidade na prevenção ou manejo das complicações de diversas doenças crônicas não transmissíveis (BRAGA e BARLETA, 2017; COSTA e ROSA, 2016; ZAPAROLLI et al., 2013).

É oportuno destacar que muitos alimentos funcionais, distribuídos amplamente na natureza, são consumidos cotidianamente na versão in natura ou inseridos em preparações culinárias regionais, e a maioria das pessoas desconhecem seu potencial benéfico à saúde (VIDAL et al., 2012). Dietas ricas em frutas, verduras, cereais integrais, leite de soja e fontes alimentares de ômega-3 assumem relevância ainda não completamente compreendida para promoção da saúde. Além disso, para que seja realizada uma alimentação saudável, todos os grupos de alimentos devem compor a dieta habitual, fracionada em diferentes refeições, que são distribuídas ao longo do dia (JAIME et al., 2015).

Ressalta-se que as refeições diárias servem não apenas como meio para ofertar alimentos que irão nutrir, otimizar a saúde e prevenir doenças, mas também, culturalmente, os momentos que envolvem a alimentação são eventos agradáveis, oportunidades de socialização e de intimidade familiar. Como consequência, a promoção da alimentação saudável também deve considerar modificações históricas importantes que 
resultaram no aumento de refeições realizadas fora do domicílio, do consumo de alimentos industrializados e refeições pré-preparadas ou prontas, em virtude da praticidade que representam em uma sociedade onde as mulheres assumiram o mercado de trabalho (STRINGHETA et al., 2007).

No Brasil, dados da Pesquisa de Orçamento Familiar de 2008/2009 estimam que 37\% do gasto mensal das famílias brasileiras urbanas é destinado à alimentação fora do domicílio (BRASIL, 2010; CAVALCANTE et al., 2017). Nesses casos, um dos principais pontos de alimentação disponíveis nos centros urbanos é o restaurante self-service (RSS), caracterizado pelo autoatendimento do cliente, que após se servir das refeições disponibilizadas, paga pela alimentação o equivalente em dinheiro ao peso do prato. A modalidade de alimentação self-service oferece, portanto, economia, rapidez e refeições regionais, representando a preferência da população urbana no horário do almoço (AVELAR e CARVALHO, 2013).

Desta forma, considerando a importância da conscientização populacional quanto às escolhas alimentares e o RSS como ambiente propício para atividades de avaliação e promoção da alimentação saudável, 0 presente estudo tem por objetivo descrever o consumo alimentar e o conhecimento sobre alimentos funcionais de usuários de restaurante self-service, localizado no centro de uma capital do nordeste brasileiro.

\section{MÉTODOS}

\section{Desenho de estudo e população}

Trata-se de um estudo descritivo de delineamento transversal, realizado em RSS, localizado no centro da cidade de Teresina, Piauí, que funciona de segunda a sexta, servindo 380 refeições diárias no horário do almoço, com preparações elaboradas e supervisionadas por nutricionista. Atende a um público diversificado, composto por bancários, funcionários e clientes do comércio local.

Participaram deste estudo 161 indivíduos, com idade entre 20 e 59 anos, de ambos os sexos, usuários do RSS pelo menos três vezes por semana para o almoço, aptos a responder aos questionários da pesquisa. Foram excluídos os indivíduos que apresentavam deficiência física e/ou psicológica que pudesse interferir na coleta e interpretação dos dados, bem como estudantes ou bacharéis em Nutrição. A aplicação dos questionários foi realizada em ambiente privativo, no próprio restaurante, após o usuário ter realizado sua refeição.

\section{Determinação da amostra}

O tamanho da amostra foi determinado por metodologia probabilística, com auxílio da planilha estatística Raosoft $^{\circledR}$, considerando o número de adultos, de ambos os sexos, residentes em Teresina, Piauí (479.390), conforme dados do censo do Instituto Brasileiro de Geografia e Estatística (BRASIL, 2010), e utilizando uma prevalência de $50 \%$ para estimar o percentual de adultos frequentadores de RSS naquela localidade. A margem de erro considerada foi de $8 \%$ e o intervalo de confiança de $95 \%$, alcançando-se um tamanho amostral mínimo de 151 indivíduos.

\section{Avaliação do conhecimento e consumo de alimentos funcionais}

Os usuários foram investigados quanto ao conhecimento da definição de alimentos funcionais, apresentando-Ihes possibilidades de respostas, das quais apenas uma estava correta (Quadro 1).

Quadro 1. Possibilidades de respostas para a definição de alimentos funcionais.

\begin{tabular}{|l|l|}
\hline \multicolumn{1}{|c|}{ DEFINIÇÕES INCORRETAS } & \multicolumn{1}{|l|}{ DEFINIÇÃo CORRETA } \\
\hline - Não sei opinar. & $\begin{array}{l}\text { São alimentos que, além } \\
\text { de nutrientes, contêm } \\
\text { substâncias capazes de } \\
\text { - São alimentos para controle exclusivo de diabetes e hipercolesterolemia. } \\
\text { - São alimentos orgânicos. }\end{array}$ \\
\hline - São saúde ou reduzir \\
\hline
\end{tabular}

Fonte: Dados da pesquisa, 2018. 
No que se refere ao consumo de alimentos funcionais, foi aplicado um Questionário de Frequência Alimentar (QFA) do International Physical Activity Questionaire, adaptado para o português por Maciel et al. (2012). Este instrumento foi elaborado com foco analítico sobre os grupos de alimentos, a fim de identificar o consumo de alimentos funcionais nos 15 dias que antecederam a avaliação. Além disso, com a finalidade de adequar o instrumento a este estudo, os alimentos listados em cada grupo alimentar foram avaliados pelos pesquisadores, quanto à ocorrência dos mesmos nas preparações oferecidas no RSS para a refeição almoço e sua utilização na culinária regional. Por este motivo, alguns alimentos com propriedades funcionais, a exemplo da linhaça e da chia, não foram incluídos no questionário alimentar e avaliados neste estudo.

Foram organizados sete grupos alimentares: (1) cereais e leguminosas, (2) óleos, oleaginosas e gorduras, (3) carnes, frutos do mar e ovos, (4) leites e derivados, (5) hortaliças, (6) frutas, (7) bebidas e especiarias. Os componentes funcionais considerados no grupo de bebidas e especiarias foram os antioxidantes, flavonoides, anticarcinogênicos, anti-inflamatórios e termogênicos. Nos demais grupos, verificou-se o consumo de ácidos graxos saturados e insaturados (carnes ovos, peixes, azeites, óleos, laticínios), prebióticos e probióticos (leites fermentados e iogurtes), fibras, vitaminas, minerais (frutas, hortaliças, cereal integral), proteína vegetal e isoflavonas (leguminosas) (Quadro 2).

Quadro 2. Organização do Questionário de Frequência Alimentar, segundo grupos alimentares e alimentos funcionais que o compõe.

\begin{tabular}{|l|l|}
\hline \multicolumn{1}{|c|}{ Grupos de alimentos } & \multicolumn{1}{c|}{ Alimentos funcionais } \\
\hline Cereais e Leguminosas & Arroz integral, milho verde, macarrão, feijão e proteína texturizada de soja. \\
\hline $\begin{array}{l}\text { Óleos, Oleaginosas e } \\
\text { Gorduras }\end{array}$ & $\begin{array}{l}\text { Óleos de soja, coco, milho e girassol, azeite de oliva, castanhas do Brasil e de } \\
\text { caju, amendoim, banha de porco, bacon, margarina e manteiga. }\end{array}$ \\
\hline $\begin{array}{l}\text { Carnes, Frutos do Mar e e } \\
\text { Ovos }\end{array}$ & $\begin{array}{l}\text { Carnes bovina, suína, ovina e de frango, fígado bovino, peixe fresco, peixe } \\
\text { enlatado, peixes conservados em sal, camarão, caranguejo, ovos fritos e } \\
\text { cozidos. }\end{array}$ \\
\hline Leites e Derivados & $\begin{array}{l}\text { Leite integral, leite semidesnatado ou desnatado, leites fermentados e } \\
\text { iogurtes, queijos brancos (ricota, minas frescal), queijos amarelos (prato e } \\
\text { mozzarella) e requeijão. }\end{array}$ \\
\hline Hortaliças & $\begin{array}{l}\text { Alface, repolho branco, repolho roxo, batata doce, batata inglesa, macaxeira, } \\
\text { pepino, tomate, cebola, couve folha, couve-flor, pimentão, abóbora, ervilha, } \\
\text { abobrinha, berinjela, cenoura, beterraba, rúcula, quiabo, vagem e azeitona. }\end{array}$ \\
\hline Frutas & $\begin{array}{l}\text { Melancia, melão, maracujá, laranja, limão, coco verde, manga, abacate, } \\
\text { mamão, banana, tamarindo, acerola, caju, cajá, abacaxi, graviola, buriti, bacuri } \\
\text { e uva. }\end{array}$ \\
\hline Bebidas e Especiarias & $\begin{array}{l}\text { Vinho tinto, suco de uva roxa, açafrão, chá verde, chá preto, chá de camomila, } \\
\text { de erva-cidreira e de limão, canela, pimenta, cominho, cúrcuma, pimenta do } \\
\text { reino, mostarda, cravo da índia, gengibre e alho. }\end{array}$ \\
\hline
\end{tabular}

Fonte: Dados da pesquisa, 2018.

A frequência do consumo alimentar foi analisada de acordo com as categorias: consumo habitual, quando o alimento foi mencionado >4 vezes/semana; não habitual, quando o consumo foi $\leq 4$ vezes/semana; raramente consumido, quando mencionado na alimentação de 1 a 3 vezes/mês, e alimento não consumido.

REAS/EJC | Vol.11(1) | DOI: https://doi.org/10.25248/reas30_2019 Página 2372 de 2379 


\section{Análise dos dados}

Os dados foram analisados por estatística descritiva, determinando-se frequências, médias e desvios padrão, com auxílio do software IBM SPSS Statistics (versão 22.0, 2013). Os resultados foram expressos em figura e tabelas.

\section{Aspectos éticos}

O estudo foi aprovado pelo Comitê de Ética em Pesquisa do Centro Universitário UNINOVAFAPI, sob registro do Certificado de Apresentação para Apreciação Ética (CAAE) ํo 32537514.9.0000.5210. Todos os participantes assinaram o Termo de Consentimento Livre e Esclarecido, elaborado de acordo com a Resolução no 466/12 do Conselho Nacional de Saúde. Foi garantida a total confidencialidade dos dados individuais e o direito de desistir da pesquisa em qualquer etapa da mesma.

\section{RESULTADOS}

A população estudada foi composta por usuários adultos, com média de idade de 38,6 $\pm 9,0$ anos, e predominância de indivíduos do sexo masculino $(57,8 \%)$, com escolaridade igual ou maior que 12 anos de estudo, equivalente ao ensino superior $(73,3 \%)$ (Tabela 1).

A definição de alimentos funcionais foi identificada corretamente por $36,6 \%(n=59)$ dos usuários avaliados, $57,8 \%(n=93)$ marcaram definições erradas e $5,6 \%(n=9)$ não souberam opinar. A distribuição percentual dos usuários, de acordo com as possibilidades de definição para alimentos funcionais, está demonstrada na Figura 1.
Tabela 1. Caracterização dos usuários de RSS, segundo sexo, faixa etária e nível de escolaridade.

\begin{tabular}{ccc}
\hline Parâmetros & N & $\%$ \\
\hline Sexo & 68 & 42,2 \\
Feminino & 93 & 57,8 \\
Masculino & & \\
Faixa Etária & 75 & 46,6 \\
20 a 39 anos & 86 & 53,4 \\
40 a 59 anos & & \\
Nível de Escolaridade & 4 & 2,5 \\
Ensino Fundamental & 39 & 29,2 \\
Ensino Médio & 118 & 73,3 \\
Ensino Superior &
\end{tabular}

Fonte: Dados da pesquisa, 2018.

Figura 1. Distribuição percentual dos usuários de RSS, segundo as possibilidades de definição para alimentos funcionais.

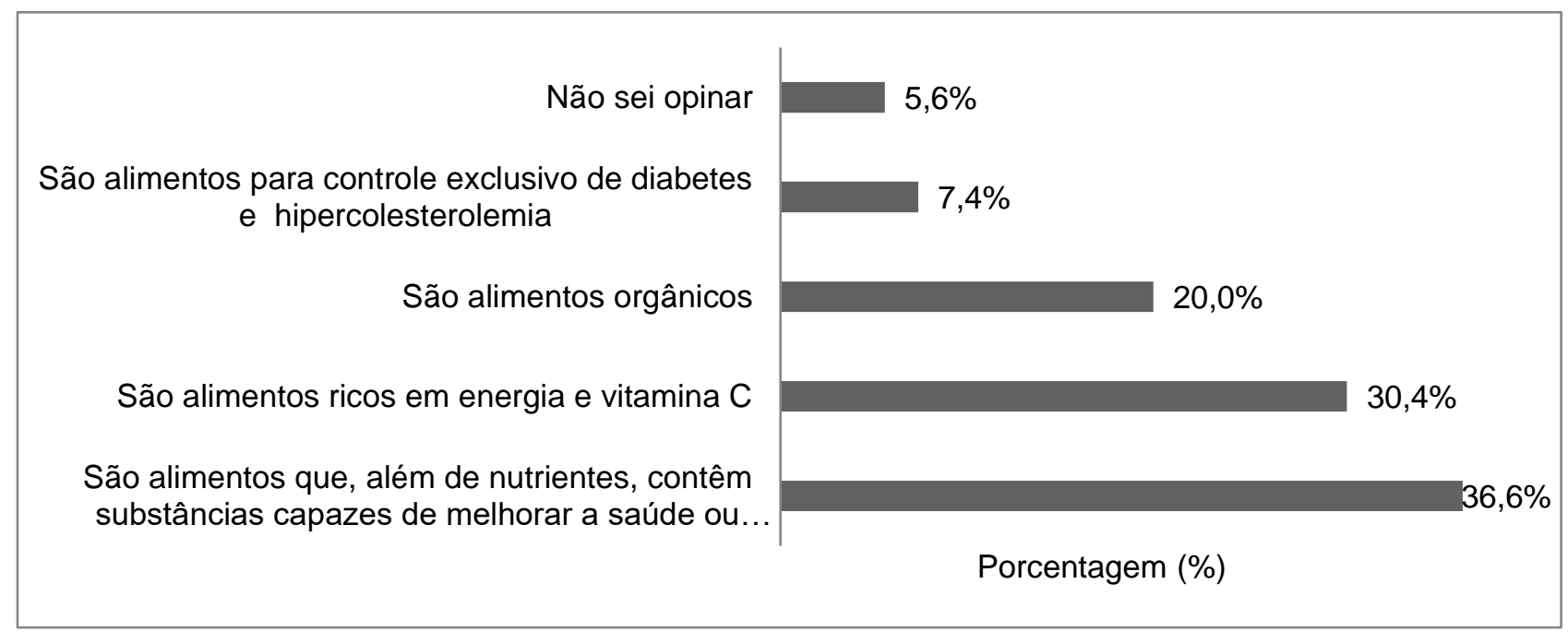

Fonte: Dados da pesquisa, 2018. 
No que se refere ao consumo alimentar, os resultados estão apresentados por grupos de alimentos, segundo frequência alimentar relatada pelos usuários de RSS. Entre os alimentos com nutrientes e/ou componentes funcionais que são consumidos habitualmente ( $>4$ vezes/semana) destacam-se o leite integral, tomate, pepino e pimentão, sendo raramente (1 a 3 vezes/mês) ou nunca consumidos: arroz integral, soja, óleo de coco, amendoim, leite desnatado, frutos do mar, queijo, repolho roxo, couve folha, berinjela, rúcula, graviola, bacuri, tamarindo e as especiarias. Alimentos funcionais consumidos não habitualmente $(\leq 4$ vezes/semana) nos diferentes grupos de alimentos, foram: castanhas de caju e do Brasil, peixes, ovos, batata inglesa, cenoura, beterraba, melancia, melão e banana. Os resultados demonstraram ainda que feijão, óleo de soja, azeite de oliva, alface e cebola são mencionados como alimentos de consumo habitual e não habitual em proporção similar entre os indivíduos pesquisados (Tabela 2).

Tabela 2. Frequência de consumo alimentar por usuários do RSS, segundo grupos de alimentos.

\begin{tabular}{|c|c|c|c|c|c|c|c|c|}
\hline \multirow{3}{*}{ Grupo de alimentos } & \multicolumn{8}{|c|}{ Frequência de consumo alimentar } \\
\hline & \multicolumn{2}{|c|}{$\begin{array}{c}\begin{array}{c}\text { Consumo } \\
\text { habitual }\end{array} \\
>4 \text { x/sem }\end{array}$} & \multicolumn{2}{|c|}{$\begin{array}{c}\begin{array}{c}\text { Consumo não } \\
\text { habitual }\end{array} \\
\leq 4 \mathrm{x} / \text { sem }^{*} \\
\end{array}$} & \multicolumn{2}{|c|}{$\begin{array}{c}\begin{array}{c}\text { Raramente } \\
\text { consumido }\end{array} \\
1 \text { a } 3 \mathrm{x} / \mathrm{m}^{\star \star}\end{array}$} & \multicolumn{2}{|c|}{$\begin{array}{c}\text { Não } \\
\text { consumido }\end{array}$} \\
\hline & $\mathbf{N}$ & $\%$ & $\mathbf{N}$ & $\%$ & $\mathbf{N}$ & $\%$ & $\mathbf{N}$ & $\%$ \\
\hline \multicolumn{9}{|c|}{ Arroz integral e leguminosas } \\
\hline Arroz integral & 3 & 1,8 & 7 & 4,3 & 54 & 33,5 & 97 & 60,2 \\
\hline Feijão & 77 & 47,8 & 78 & 48,4 & 1 & 0,6 & 5 & 3,1 \\
\hline Soja & 1 & 0,6 & 1 & 0,6 & 44 & 27,3 & 115 & 71,4 \\
\hline \multicolumn{9}{|c|}{ Óleos, oleaginosas e gorduras } \\
\hline Margarina & 77 & 47,8 & 54 & 33,5 & 10 & 6,2 & 20 & 12,4 \\
\hline Manteiga & 29 & 18 & 63 & 39,1 & 17 & 10,5 & 52 & 32,2 \\
\hline Óleo de coco & 30 & 18,6 & 26 & 16,1 & 8 & 4,9 & 97 & 60,2 \\
\hline Óleo de soja & 44 & 27,3 & 40 & 24,8 & 16 & 9,9 & 61 & 37,8 \\
\hline Azeite de oliva & 41 & 25,4 & 41 & 25,4 & 38 & 23,6 & 41 & 25,4 \\
\hline Castanha de caju & 6 & 3,7 & 35 & 21,7 & 60 & 37,2 & 60 & 37,2 \\
\hline Castanha do Brasil & 12 & 7,4 & 36 & 22,3 & 63 & 39,1 & 50 & 31 \\
\hline Amendoim & 5 & 3,1 & 22 & 13,6 & 44 & 27,3 & 90 & 55,9 \\
\hline \multicolumn{9}{|c|}{ Carnes, frutos do mar e ovos } \\
\hline Carne bovina & 33 & 20,4 & 96 & 59,6 & 16 & 9,9 & 16 & 9,9 \\
\hline Carne ovina & 4 & 2,4 & 62 & 38,5 & 29 & 18 & 66 & 40,9 \\
\hline Carne suína & 11 & 6,8 & 68 & 42,2 & 29 & 18 & 53 & 32,9 \\
\hline Frango & 49 & 30,4 & 107 & 66,4 & 1 & 0,6 & 4 & 2,4 \\
\hline Miúdos & 6 & 3,7 & 46 & 28,5 & 63 & 39,1 & 46 & 28,5 \\
\hline Peixe de água doce & 2 & 1,2 & 64 & 39,7 & 51 & 31,6 & 44 & 27,3 \\
\hline Peixe de água salgada & 1 & 0,6 & 64 & 39,7 & 47 & 27,3 & 49 & 30,4 \\
\hline Camarão & 1 & 0,6 & 22 & 13,6 & 47 & 27,3 & 91 & 56,4 \\
\hline Caranguejo & 0 & 0 & 14 & 8,6 & 47 & 27,3 & 100 & 62,1 \\
\hline Peixes conservado (sal) & 1 & 0,6 & 20 & 12,4 & 33 & 20,4 & 106 & 65,8 \\
\hline Ovos & 23 & 14,2 & 115 & 71,4 & 15 & 9,3 & 8 & 4,9 \\
\hline \multicolumn{9}{|l|}{ Leites e derivados } \\
\hline Leite integral & 60 & 37,2 & 42 & 26 & 10 & 6,2 & 49 & 30,4 \\
\hline Leite semidesnatado & 14 & 8,6 & 19 & 11,8 & 15 & 9,3 & 108 & 67 \\
\hline Leite desnatado & 8 & 4,9 & 37 & 22,9 & 8 & 4,9 & 113 & 70,1 \\
\hline logurte Integral & 4 & 2,4 & 45 & 27,9 & 14 & 8,6 & 97 & 60,2 \\
\hline Queijo & 0 & 0 & 42 & 26 & 15 & 9,3 & 104 & 64,5 \\
\hline
\end{tabular}




\begin{tabular}{|c|c|c|c|c|c|c|c|c|}
\hline \multicolumn{9}{|l|}{ Continuação Tabela 2} \\
\hline \multirow{4}{*}{ Grupo de alimentos } & \multicolumn{8}{|c|}{ Frequência de consumo alimentar } \\
\hline & \multirow{2}{*}{\multicolumn{2}{|c|}{$\begin{array}{c}\begin{array}{c}\text { Consumo } \\
\text { habitual }\end{array} \\
>4 \mathrm{x} / \text { sem }^{*}\end{array}$}} & \multirow{2}{*}{\multicolumn{2}{|c|}{$\begin{array}{c}\begin{array}{c}\text { Consumo não } \\
\text { habitual }\end{array} \\
\leq 4 \mathrm{x} / \mathrm{sem}^{*} \\
\end{array}$}} & \multirow{2}{*}{\multicolumn{2}{|c|}{$\begin{array}{c}\begin{array}{l}\text { Raramente } \\
\text { consumido }\end{array} \\
1 \text { a } 3 \times / m^{\star \star} \\
\end{array}$}} & \multirow{2}{*}{\multicolumn{2}{|c|}{$\begin{array}{c}\text { Não } \\
\text { consumido }\end{array}$}} \\
\hline & & & & & & & & \\
\hline & $\mathbf{N}$ & $\%$ & $\mathbf{N}$ & $\%$ & $\mathbf{N}$ & $\%$ & $\mathbf{N}$ & $\%$ \\
\hline \multicolumn{9}{|l|}{ Hortaliças } \\
\hline Alface & 57 & 35,4 & 57 & 35,4 & 30 & 18,6 & 17 & 10,5 \\
\hline Repolho branco & 33 & 20,4 & 57 & 35,4 & 16 & 9,9 & 55 & 34,1 \\
\hline Repolho roxo & 12 & 7,4 & 38 & 23,6 & 20 & 12,4 & 91 & 56,5 \\
\hline Batata doce & 19 & 11,8 & 56 & 34,7 & 32 & 19,8 & 54 & 33,5 \\
\hline Batata inglesa & 10 & 6,2 & 116 & 72 & 24 & 14,9 & 11 & 6,8 \\
\hline Pepino & 46 & 28,5 & 73 & 45,3 & 19 & 11,8 & 23 & 14,2 \\
\hline Tomate & 54 & 33,5 & 76 & 47,2 & 5 & 3,1 & 26 & 16,1 \\
\hline Cebola & 49 & 30,4 & 45 & 27,9 & 11 & 6,8 & 56 & 34,7 \\
\hline Couve folha & 6 & 3,7 & 50 & 31 & 9 & 5,5 & 96 & 59,6 \\
\hline Couve-flor & 10 & 6,2 & 44 & 27,3 & 26 & 16,1 & 81 & 50,3 \\
\hline Pimentão & 49 & 30,4 & 69 & 42,8 & 16 & 9,9 & 27 & 16,7 \\
\hline Abobrinha & 10 & 6,2 & 64 & 39,7 & 28 & 17,3 & 59 & 36,6 \\
\hline Berinjela & 4 & 2,4 & 14 & 8,6 & 22 & 13,6 & 121 & 75,1 \\
\hline Cenoura & 22 & 13,6 & 113 & 70,1 & 14 & 8,6 & 12 & 7,4 \\
\hline Beterraba & 23 & 14,2 & 100 & 62,1 & 26 & 16,1 & 12 & 7,4 \\
\hline Rúcula & 3 & 1,8 & 17 & 10,5 & 25 & 15,5 & 116 & 72 \\
\hline Vagem & 11 & 6,8 & 41 & 25,4 & 16 & 9,9 & 93 & 57,7 \\
\hline \multicolumn{9}{|l|}{ Frutas } \\
\hline Melancia & 17 & 10,5 & 81 & 50,3 & 40 & 24,8 & 23 & 14,2 \\
\hline Melão & 10 & 6,2 & 102 & 63,3 & 26 & 16,1 & 23 & 14,2 \\
\hline Maracujá & 18 & 11,1 & 79 & 49 & 23 & 14,2 & 40 & 24,8 \\
\hline Laranja & 30 & 18,6 & 75 & 46,5 & 13 & 8 & 42 & 26 \\
\hline Limão & 24 & 14,9 & 47 & 29,1 & 31 & 19,2 & 59 & 36,6 \\
\hline Coco verde & 7 & 4,3 & 64 & 39,7 & 46 & 28,5 & 44 & 27,3 \\
\hline Manga & 19 & 11,8 & 68 & 42,2 & 27 & 16,7 & 47 & 29,1 \\
\hline Abacate & 0 & 0 & 35 & 21,7 & 49 & 30,4 & 77 & 47,8 \\
\hline Mamão & 9 & 5,5 & 73 & 45,3 & 25 & 15,5 & 54 & 33,5 \\
\hline Banana & 50 & 31 & 80 & 49,6 & 6 & 3,7 & 25 & 15,5 \\
\hline Tamarindo & 0 & 0 & 25 & 15,5 & 46 & 28,5 & 90 & 55,9 \\
\hline Acerola & 4 & 2,4 & 64 & 39,7 & 36 & 22,3 & 57 & 35,4 \\
\hline Caju & 4 & 2,4 & 66 & 40,9 & 53 & 32,9 & 38 & 23,6 \\
\hline Cajá & 4 & 2,4 & 66 & 40,9 & 40 & 24,8 & 51 & 31,6 \\
\hline Abacaxi & 6 & 3,7 & 58 & 36 & 29 & 18 & 68 & 42,2 \\
\hline Graviola & 0 & 0 & 13 & 8 & 19 & 11,8 & 129 & 80,1 \\
\hline Buriti & 1 & 0,6 & 35 & 21,7 & 25 & 15,5 & 100 & 62,1 \\
\hline Uva roxa & 25 & 15,5 & 70 & 43,4 & 25 & 15,5 & 41 & 25,4 \\
\hline
\end{tabular}




\begin{tabular}{|c|c|c|c|c|c|c|c|c|}
\hline \multicolumn{9}{|l|}{ Continuação Tabela 2} \\
\hline \multirow{3}{*}{ Grupo de alimentos } & \multicolumn{8}{|c|}{ Frequência de consumo alimentar } \\
\hline & \multicolumn{2}{|c|}{$\begin{array}{c}\text { Consumo } \\
\text { habitual } \\
>4 \text { x/sem }{ }^{\star}\end{array}$} & \multicolumn{2}{|c|}{$\begin{array}{c}\text { Consumo não } \\
\text { habitual } \\
\leq 4 \mathrm{x} / \mathrm{sem}^{*}\end{array}$} & \multicolumn{2}{|c|}{$\begin{array}{c}\begin{array}{l}\text { Raramente } \\
\text { consumido }\end{array} \\
1 \text { a } 3 \mathrm{x} / \mathrm{m}^{\star *}\end{array}$} & \multicolumn{2}{|c|}{$\begin{array}{c}\text { Não } \\
\text { consumido }\end{array}$} \\
\hline & $\mathbf{N}$ & $\%$ & $\mathbf{N}$ & $\%$ & $\mathbf{N}$ & $\%$ & $\mathbf{N}$ & $\%$ \\
\hline \multicolumn{9}{|l|}{ Bebidas e especiarias } \\
\hline Chá verde & 1 & 0,6 & 15 & 9,3 & 2 & 1,2 & 143 & 88,8 \\
\hline Chá preto & 0 & 0 & 3 & 1,8 & 1 & 0,6 & 157 & 97,5 \\
\hline Chá de erva cidreira & 5 & 3,1 & 24 & 14,9 & 8 & 4,9 & 124 & 77 \\
\hline Chá de camomila & 2 & 1,2 & 20 & 12,4 & 6 & 3,7 & 133 & 82,6 \\
\hline Chá de limão & 1 & 0,6 & 15 & 9,3 & 8 & 4,9 & 137 & 85 \\
\hline Açafrão & 0 & 0 & 0 & 0 & 4 & 2,4 & 157 & 97,5 \\
\hline Canela & 0 & 0 & 10 & 6,2 & 9 & 5,5 & 142 & 88,1 \\
\hline Pimenta & 13 & 8 & 13 & 8 & 9 & 5,5 & 126 & 78,2 \\
\hline Cominho & 9 & 5,5 & 13 & 8 & 8 & 4,9 & 131 & 81,3 \\
\hline Cúrcuma & 4 & 2,4 & 6 & 3,7 & 2 & 1,24 & 149 & 92,5 \\
\hline Pimenta do reino & 21 & 13 & 17 & 10,5 & 3 & 1,8 & 120 & 74,5 \\
\hline Mostarda & 1 & 0,6 & 5 & 3,1 & 1 & 0,6 & 152 & 94,4 \\
\hline Cravo da índia & 0 & 0 & 7 & 4,3 & 3 & 1,8 & 153 & 95 \\
\hline Gengibre & 0 & 0 & 1 & 0,6 & 3 & 1,8 & 151 & 93,7 \\
\hline Alho & 32 & 19,8 & 22 & 13,6 & 2 & 1,2 & 105 & 65,2 \\
\hline Alecrim & 2 & 1,2 & 5 & 3,1 & 1 & 0,6 & 153 & 95 \\
\hline
\end{tabular}

Fonte: Dados da pesquisa, 2018.

Além dos resultados mencionados, a tabela 2 permite a análise detalhada da frequência de consumo, considerando cada grupo de alimentos. Assim, observou-se no grupo das carnes, que $30,4 \%$ dos indivíduos pesquisados indicaram consumo $>4$ vezes/semana de frango, e para 59,6\% a carne bovina não estava incluída na alimentação habitual. Miúdos foram indicados como raramente consumidos por $39,1 \%$ dos indivíduos.

No grupo de leites e derivados, o alimento mais frequentemente consumido foi o leite integral, presente $>4$ vezes/semana na dieta de $37,2 \%$ dos indivíduos pesquisados. Leite desnatado e iogurte integral foram mencionados com consumo $>4$ vezes/semana por apenas $4,9 \%$ e $2,4 \%$ dos indivíduos, respectivamente, enquanto o queijo não foi indicado por nenhum participante.

Entre as hortaliças, repolho branco e batata doce foram mencionados com consumo semanal, embora $\leq 4$ vezes, respectivamente por $35,4 \%$ e $34,2 \%$ dos indivíduos pesquisados. No grupo das frutas, destacaram-se a melancia $(50,3 \%)$, maracujá $(49 \%)$, laranja $(46,5 \%)$, coco verde $(39,7 \%)$, manga $(42,2 \%)$, banana $(45,3 \%)$, acerola $(39,7 \%)$, caju $(40,9 \%)$ e uva roxa $(43,4 \%)$, como alimentos consumidos semanalmente, embora com frequência $\leq 4$ vezes nesse período.

No que se refere às especiarias, o alho é o mais consumido, estando presente na alimentação habitual de $19,8 \%$ dos indivíduos, com frequência $>4$ vezes por semana, e de 13,6\% com frequência $\leq 4$ vezes por semana. Por outro lado, açafrão, gengibre, cravo da índia, mostarda, alecrim e cúrcuma foram indicados, respectivamente, por $97,5 \%, 93,7 \%, 95 \%, 94,4 \%, 95 \%$ e $92,5 \%$ dos indivíduos, como nunca consumido. Ressalta-se que os chás mais consumidos pela população investigada foram os de erva cidreira $(14,9 \%)$ e de camomila $(12,4 \%)$, em uma frequência semanal $\leq 4$ vezes. Os chás verde e preto foram indicados como nunca consumidos por $88,8 \%$ e $97,5 \%$ dos indivíduos pesquisados, respectivamente. 


\section{DISCUSSÃO}

A industrialização e consequente urbanização brasileira promoveram modificações no estilo de vida da população, tornando frequente a alimentação fora do lar e atividades sedentárias (CLARO et al., 2015). Segundo a Pesquisa de Orçamentos Familiares (POF) de 2008/2009, do Instituto Brasileiro de Geografia e Estatística (BRASIL, 2010), os gastos com alimentação no Norte e Nordeste do país representam cerca de $25 \%$ da renda mensal familiar, enquanto para as outras regiões, o mesmo gasto representa, em média, $18,2 \%$ do total.

Uma parcela dessa despesa pode ser atribuída ao aumento na frequência de refeições realizadas fora do domicílio, que decorre, principalmente, do deslocamento das mulheres do ambiente doméstico para fortalecer a classe laboral, bem como da distância entre os ambientes doméstico e profissional e da escassez de tempo para realização das refeições, impossibilitando o retorno ao domicílio e o preparo dos alimentos (SCHLIDWEIN e KASSOUF, 2007). Essa alteração social e econômica na família brasileira é demonstrada no presente estudo, tendo em vista que quase a metade dos frequentadores de RSS entrevistados é do sexo feminino, destacando-se que todos os participantes são adultos e, portanto, economicamente ativos.

A presença das mulheres no mercado de trabalho e o aumento populacional influenciaram significativamente o padrão de consumo alimentar brasileiro, modificado de uma dieta tradicional para uma alimentação que exige pouca dedicação ao preparo dos alimentos, substituindo-os na versão natural e em preparações caseiras, por industrializados, comercializados em restaurantes, prontos ou semiprontos para 0 consumo (LELIS et al., 2012). Desta forma, o padrão alimentar contemporâneo, adotado gradativamente nos últimos 20 anos, é caracterizado pelo elevado consumo de alimentos ricos em carboidratos simples, sódio e gorduras saturadas, bem como por reduzida ingestão de alimentos fontes de fibras, ácidos graxos insaturados, vitaminas e minerais (FIGUEIRÊDO-REINALDO et al., 2015).

Associado ao sedentarismo, o padrão alimentar contemporâneo contribuiu para a modificação do perfil de morbimortalidade da população. Problemas de saúde pública, antes relacionados às carências alimentares e doenças infectocontagiosas, foram substituídos por afecções crônicas, como obesidade, doenças cardiovasculares, dislipidemias, cânceres e diabetes, que decorrem, em parte, de uma alimentação sem equilíbrio nutricional. Como consequência, nas últimas décadas tem sido observado o aumento crescente da prevalência dessas doenças crônicas entre crianças e adolescentes (MALTA et al., 2013).

Nesse cenário, a escolaridade, o conhecimento e a capacidade de fazer escolhas alimentares destacamse entre os fatores determinantes da qualidade da alimentação (ESTIMA et al., 2009; ROSSI et al., 2008). No presente estudo, entretanto, a escolaridade não parece estar relacionada às escolhas alimentares como meio de beneficiar a saúde e reduzir o risco de doenças crônicas. Ao serem questionados sobre a definição de alimentos funcionais, a maioria dos participantes os identificaram como "alimentos orgânicos", "ricos em energia e vitamina C", "alimentos para controle exclusivo do diabetes e colesterol elevado" ou não souberam opinar a respeito. Esses resultados são semelhantes aos encontrados por Campos et al. (2016), em estudo conduzido com frequentadores de self-service de Teresina, Piauí, e podem ser justificados pelo acesso às informações errôneas e/ou confusas difundidas pela mídia.

No que se refere à promoção da saúde por meio da dieta, o consumo de alimentos funcionais compreende uma abordagem emergente para prevenção de doenças crônicas, a partir de efeitos fisiológicos benéficos induzidos por ingredientes ativos contidos nos alimentos, sejam ou não nutrientes e/ou fontes de energia.

Estudos demonstram que o risco para doenças cardiovasculares pode ser $30 \%$ menor a partir de modificações na dieta, bem como indicam que os fatores dietéticos representam cerca de 20 a $30 \%$ das causas de câncer, sendo somente superado pelo tabaco, como fator de risco prevenível (NEUMANN et al., 2007). Esses dados são corroborados pela Organização Mundial de Saúde (2011), segundo a qual o consumo insuficiente de frutas, legumes e verduras é fator determinante para 2,7 milhões de mortes anuais decorrentes de doenças crônicas não transmissíveis. 
No estado Piauí, onde foi conduzido este estudo, em 2015, as principais causas de óbito registradas foram por doenças crônicas não transmissíveis, com taxa por 100.000 habitantes, de 85 por doenças cardiovasculares, 59,3 por neoplasias, 43,6 por hipertensão arterial sistêmica e 41,7 por diabetes (BRASIL, 2015). Assim, os resultados do presente estudo, referente ao consumo alimentar habitual da população investigada, ratifica o perfil de morbimortalidade descrito, pois se identificou baixa ingestão semanal de frutas, hortaliças, cereal integral, leguminosas, óleos insaturados, chás e especiarias, considerados alimentos funcionais por serem fontes de vitaminas, minerais e componentes bioativos. Este consumo insuficiente é semelhante ao encontrado por Brito et al. (2012) em estudo que investigou o consumo alimentar anterior ao diagnóstico em pacientes com câncer.

Quanto aos compostos bioativos, é importante mencionar que seu papel na prevenção de doenças crônicas ocorre em virtude das atividades antioxidante, anti-inflamatória e anticarcinogênica exercidas no organismo, com impacto metabólico. Como exemplos, destacam-se o resveratrol (uva roxa, fruta ou suco), licopeno (tomate), organossulforetos (alho e crucíferos), catequinas (chá verde), gingerol (gengibre) e curcumina (açafrão), pois são capazes de modular a expressão de genes codificadores de proteínas envolvidas em mecanismos de defesa contra processos degenerativos (DAIMIEL et al., 2012). Neste estudo, todos os alimentos fonte destes compostos bioativos não são consumidos regularmente pela maioria dos participantes, tendo sido relatada frequência de consumo inferior a quatro vezes por semana.

No que se refere ao consumo de laticínios, grupo alimentar que reúne as principais fontes de cálcio, demonstrou-se ingestão habitual de leite integral por apenas $37,2 \%$ dos entrevistados. A versão desnatada ou alimentos derivados, a exemplo de queijos e iogurtes, os entrevistados relataram frequência não habitual ou nunca consumido. Estes achados são concordantes com os demonstrados por Carvalho e Rocha (2011), bem como ratificam os valores médios de ingestão de cálcio pela população brasileira, que é inferior a $400 \mathrm{mg}$ do mineral por dia (LANZILLOTTI et al., 2013). Ressalta-se que, a ingestão insuficiente de cálcio é indicador de risco para doenças osteometabólicas relacionadas à carência desse mineral, como osteoporose, osteomalácia e osteopenia, prevalentes na população feminina.

Além disso, é relevante destacar o consumo não habitual de óleos vegetais, azeite de oliva e peixes, em concordância com os resultados demonstrados por Lima et al. (2015). Estes alimentos são importantes pelo teor de ômega 3 e outros ácidos graxos insaturados, que modulam a resposta inflamatória à dieta, pois estimulam a síntese de enzimas, a expressão gênica de proteínas anti-inflamatórias e anti-apoptóticas, além de reduzir a expressão de marcadores pró-inflamatórios e pró-apoptóticos no hipotálamo. Estes ácidos graxos exercem atividade nas vias de sinalização, transdução de sinais e proliferação celular, com impacto na síntese de marcadores imunomodulatórios da inflamação, cicatrização e estresse oxidativo (OLIVEIRA e PINHEIRO, 2013). Ressalta-se, portanto, que dietas habituais com baixo teor de ácidos graxos insaturados favorecem um estado clínico de inflamação crônica de baixo grau, considerada base fisiopatológica comum a várias doenças crônicas não transmissíveis (DCNT), entre as quais o câncer e as doenças cardiovasculares.

Nesse contexto, considerando que as doenças cardiovasculares são a principal causa de morte no Brasil e no mundo, realizar intervenções educativas que visem estimular o consumo de alimentos funcionais poderá contribuir para melhoria dos hábitos alimentares e saúde dos indivíduos (MS, 2011; VIDAL et al., 2012).

\section{CONCLUSÃO}

Os resultados obtidos permitem concluir que predomina o conhecimento insuficiente quanto à definição correta de alimentos funcionais pelos usuários de restaurante self-service avaliados, sugerindo-se que não há influência da escolaridade sobre o mesmo. Apesar da elevada escolaridade, os usuários demonstraram não compreender completamente as informações que possuem sobre alimentos funcionais. Desta forma, apesar da disponibilidade e da facilidade de acesso a alimentos funcionais e preparações que os contenham, estes não integram o hábito alimentar da maioria dos participantes. A dieta habitual demonstrada por este estudo segue o padrão alimentar contemporâneo, pobre em frutas e verduras, porém rica em açúcares e lipídeos, sendo, portanto, favorável ao desenvolvimento de doenças crônicas não transmissíveis. 


\section{AGRADECIMENTOS}

Os autores são gratos ao Restaurante Máximo Self-Service, localizado no centro comercial da cidade de Teresina, Piauí, Brasil; a todos os funcionários que contribuíram para a realização da coleta de dados e a todos os usuários que aceitaram, voluntariamente, participar deste estudo.

\section{CONFLITO DE INTERESSES}

Os autores declaram que não existem conflitos de interesses.

\section{REFERÊNCIAS}

1. AVELAR AE, CARVALHO DR. Hábitos alimentares fora do lar: um estudo de caso em Lavras-MG. Organizações Rurais Agroindustriais, 2013; 15(1): 137-152.

2. BRAGA AAA, BARLETA VCN. Alimento funcional: uma nova abordagem terapêutica das dislipidemias como prevenção da doença aterosclerótica. Caderno UniFOA, 2017; 2(3): 100-120.

3. BRASIL. Instituto Brasileiro de Geografia e Estatística. Pesquisa de Orçamentos Familiares 2008-2009: despesas, rendimentos e condições de vida. Rio de Janeiro: IBGE; 2010.

4. BRASIL. Portaria n.ํ 398, de 30 de abril de 1999. Aprova o regulamento técnico que estabelece as diretrizes básicas para análise e comprovação de propriedades funcionais e/ou de saúde alegadas em rotulagem de alimentos. [acesso em: 2018 Jan 08].

5. BRASIL. Ministério da Saúde. Caderno de Informações de Saúde: informações gerais município de Teresina 2015. [site da Internet] [acesso em: 2018 Jan 22].

6. BRITO LF, SILVA LS, FERNANDES DD et al. Perfil nutricional de paciente com câncer assistidos pela casa de acolhimento ao paciente oncológico do sudoeste da Bahia. Revista Brasileira de Cancerologia, 2012; 58(2): 163-171.

7. CAMPOS CMF, ARAUJO MAM, MOREIRA-ARAÚJO RSR. Consumo de alimentos funcionais por usuários de restaurantes self-service. Higiene Alimentar, 2016; 30: 34-37.

8. CARVALHO EO, ROCHA EF. Consumo alimentar de população adulta residente em área rural da cidade de Ibatiba (ES, Brasil). Ciência \& Saúde Coletiva, 2016; 16(1): 179-185.

9. CAVALCANTE JB, MOREIRA TMV, MOTA CC et al. Consumo de energia e nutrientes de acordo com o consumo de alimentos fora de casa na região Nordeste: uma análise do Inquérito Alimentar Nacional, 2008-2009. Revista Brasileira de Epidemiologia, 2017; 20(1):115-123.

10. CLARO RM, SANTOS MAS, OLIVEIRA TP et al. Consumo de alimentos não saudáveis relacionados a doenças crônicas não transmissíveis no Brasil: Pesquisa Nacional de Saúde, 2013. Epidemiologia e Serviços de Saúde, 2015; 24(2): 257-265.

11. COSTA NMB, ROSA COB. Alimentos funcionais: componentes bioativos e efeitos fisiológicos. $2^{\mathrm{a}}$ ed. Rubio. Rio de J., 2016. 504 p.

12. DAIMIEL L, VASGAS T, MOLINA AR. Nutritional genomics for the characterization of the effect of bioactive molecules in lipid metabolism and related pathways. Electrophoresis, 2012; 33(15): 2266-2289.

13. ESTIMA CCP, PHILIPPI ST, ALVARENGA MS. Fatores determinantes de consumo alimentar: por que os indivíduos comem o que comem? Revista Brasileira de Nutrição Clinica, 2009; 24(4): 263-268.

14. FIGUEIRÉDO-REINALDO ED, DA SILVA MRF, NARDOTO GB et al. Mudanças de hábitos alimentares em comunidades rurais do semiárido da região Nordeste do Brasil. Interciência, 2015; 40(5): 330-336.

15. JAIME PC, STOPA SR, OLIVEIRA TP et al. Prevalência e distribuição sociodemográfica de marcadores de alimentação saudável, Pesquisa Nacional de Saúde, Brasil 2013. Epidemiologia e Serviços de Saúde, 2015; 24(2): 267-276.

16. LANZILLOTTI HS, LANZILLOTTI RS, TROTTE APR et al. Osteoporose em mulheres na pós-menopausa: cálcio dietético e outros fatores de risco. Revista de Nutrição, 2003; 2(16): 181-193.

17. LELIS CT, TEIXEIRA KMD, SILVA NM. A inserção feminina no mercado de trabalho e suas implicações para os hábitos alimentares da mulher e de sua família. Saúde em Debate, 2012; 36(95): 523-532.

18. LIMA GS, PORTO KAO, SOUZA TKM et al. Avaliação do estado nutricional e consumo alimentar de alcoolistas atendidos em um centro de reabilitação de Caruaru-PE, Brasil. Nutrición Clinica y Dietética Hospitalaria, 2015; 35(2): 16-25.

19. MACIEL ES, SONATI JG, MODENEZE DM et al. Consumo alimentar, estado nutricional e nível de atividade física em comunidade universitária brasileira. Revista de Nutrição, 2012; 25(6): 707-718.

20. MALTA DC, ISER BPM, CLARO RM et al. Prevalência de fatores de risco e proteção para doenças crônicas não transmissíveis em adultos: estudo transversal, Brasil, 2011. Epidemiologia e Serviços de Saúde, 2013; 22(3): 423-434.

21. MINISTÉRIO DA SAÚDE (BR). Secretaria de Vigilância em Saúde. Departamento de Análise de Situação de Saúde. Plano de ações estratégicas para o enfrentamento das doenças crônicas não transmissíveis (DCNT) no Brasil 2011-2022. Brasília: Ministério da Saúde; 2011.

22. NEUMANN AICP, MARTINS IG, MARCOPITO LF et al. Padrões alimentares associados a fatores de risco para doenças cardiovasculares entre residentes de um município brasileiro. Revista Panamericana de Salud Pública, 2007; 22(5): 329-339.

23. OLIVEIRA MLM, NUNES-PINHEIRO DCS. Biomarcadores celulares e moleculares envolvidos na resposta imune-inflamatória modulada por ácidos graxos insaturados. Acta Veterinária Brasílica, 2013; 7(2): 113-124.

24. ROSSI A, MOREIRA EAM, RAUEN MS. Determinantes do comportamento alimentar: uma revisão com enfoque na família. Revista de Nutrição, 2008; 21(6): 739-748.

25. SCHLINDWEIN MM, KASSOUF AL. Influência do custo de oportunidade do tempo da mulher sobre o padrão de consumo alimentar no Brasil. Pesquisa e Planejamento Econômico, 2007; 37(3): 489-520.

26. STRINGHETA PC, OLIVEIRA TT, GOMES RC et al. Políticas de saúde e alegações de propriedades funcionais e de saúde para alimentos no Brasil. Revista Brasileira de Ciências Farmacêuticas, 2007; 43(2): 181-194.

27. WORLD HEALTH ORGANIZATION. Global status report on non communicable diseases 2010 [Internet]. Geneva: World Health Organization; 2011.

28. VIDAL AM, DIAS DO, MARTINS ESM et al. A ingestão de alimentos funcionais e sua contribuição para a diminuição de doenças. Ciências Biológicas e da Saúde, 2012; 1(15): 43-52.

29. ZAPAROLLI MR, NASCIMENTO NC, BAPTISTA DR et al. Alimentos funcionais no manejo da diabetes mellitus. Revista de Ciências da Saúde, 2013; 6(1): 12-17. 